\title{
CARACTERIZAÇÃO E EXTRAÇÃO DAS FEIÇÕES ASSOCIADAS AOS SOLOS LATERÍTICOS, ATRAVÉS DO SENSORIAMENTO REMOTO E DAS TÉCNICAS DE PROCESSAMENTO DE IMAGENS APLICADAS ÀS IMAGENS DE SATÉLITE
}

\author{
Edilberto Bezerra de Sousa ${ }^{1,2}$, Ana Lúcia Bezerra Candeias ${ }^{3}$ e Eugênio Vertamatti ${ }^{4}$
}

RESUMO

\begin{abstract}
Este trabalho apresenta uma metodologia para a caracterização e extração das feições associadas aos solos lateríticos, baseada nos estudos de Sensoriamento Remoto e em técnicas de processamento de imagens. A área teste está localizada no Estado de São Paulo, próximo à cidade de Campinas, a qual possui várias classes de latossolo. Tem-se o mapa de solo (1:50.000) desta região e as imagens TM-LANDSAT de um período seco, quando a superfície do solo está quase totalmente exposta. Técnicas de processamento de imagens, tal como restauração linear de contraste, stretching, transformação IHS, atributo de cor e filltro de fechamento e abertura, foram aplicadas para auxiliar na caracterização e extração das feições de tais solos. O sistema de informação geográfica "SPRING" e a linguagem LEGAL foram usados na implementação da metodologia. A metodologia proposta mostrou-se satisfatória no processo de caracterização e extração das feições desses solos.
\end{abstract}

Palavras-chave: solos, sensoriamento remoto, processamento de imagem, morfologia matemática

\author{
CHARACTERIZATION AND EXTRACTION OF LATERITIC SOIL \\ FEATURES BY REMOTE SENSING AND IMAGE PROCESSING TECHNIQUES \\ APPLIED TO SATELLITE IMAGES
}

\begin{abstract}
This paper presents a lateritic soil features identification based on remote sensing and image processing techniques. The test area is located in São Paulo state, near Campinas town, where the soil is composed of high percentage of iron. Soil map $(1: 50,000)$ of this region as well as TM-LANDSAT image of dry period, when the soil surface is nearly exposed, were available. Digital image processing techniques such as restoration linear contrast, stretching, I H S transformation, color attribute and closing opening filter were applied aiming to extract lateritic soils. SPRING platform and LEGAL language are used to implement the methodology.
\end{abstract}

Key words: soils, remote sensing, image processing, mathematical morphology

\section{INTRODUÇÃO}

Nos dias atuais, existe um consenso de que não é possível o desenvolvimento sistemático de um país sem que haja informações adequadas, precisas e sinópticas acerca da natureza, quantidade e distribuição dos seus recursos naturais.

Neste sentido, o conhecimento dos solos quanto às suas características lísicas, químicas e distribuição na superfície terrestre, é indispensável para o planejamento agrícola/ambiental, grandes obras de engenharia, obras viárias etc.

\footnotetext{
${ }_{1}$ Prolessor Assistente; DEAg/UFPb; Universidade Federal da Paraíba, Av. Aprígio Veloso 882, Bodocongó, 58109-970, Campina Grande, PB, Brasil. E-Mail: edil@) lmrs.br, Fone: (083) 3332355, Fax: (083) 3332035.

2 Aluno de Doutorado do Curso de Engenharia de Infra - Estrutura Aeronáutica do Instituto Tecnológico de Aeronáutica, edil@inlra.ita.br.

${ }^{3}$ Professora Adjunto; DECart/UFPE; Universidade Federal de Pernambuco; Av. Prof. Morais Rego 1235, Cidade Universitária, 50670-901, Recile, PE, Brasil. Fone: (081) 2718235

${ }^{4}$ Professor Titular; ITA; Instituto Tecnológico de Aeronáutica, Divisão de Infra-Estrutura Aeronáutica, Centro Técnico Aeroespacial-Campo Monte Negro, 12210-970, São José dos Campos - SP, verta@inlra.ita.cta.br.
} 
Os levantamentos de solo através das técnicas de sensoriamento remolo, no Brasil, remontam a 1969 , com a Missão 96, realizada pela National Aeronautic and Space Administration (NASA) através de convênio com institutos de pesquisa e ensino do país (INPE, IAC, DNPM, USP); entretanto, com o desenvolvimento tecnológico, novos sistemas sensores foram desenvolvidos (Landsat, SPOT, JERS, AVIRIS).

Com a utilização de softwares de processamento de imagens e com dados de sensores, como os citados acima, tornase possível estudar os solos em uma faixa mais ampla do espectro eletromagnético, associar cores e tratar esses dados em sistemas computacionais, de forma mais rápida, gerando uma série de produtos que podem ser utilizados como suporte no levantamento de solos.

Desta forma, este trabalho apresenta uma metodologia através do uso do sensoriamento remoto e das técnicas de processamento de imagens, que permite auxiliar na caracterização e extração das feições associadas aos solos laterílicos (Latossolos, Podzólicos, Terra Roxa) solos estes caracterizados pelo maior ou menor teor de ferro, auxiliando, assim, no processo de levantamento de solos de uma área.

\section{METODOLOGIA}

Para o desenvolvimento deste trabalho foram utilizadas imagens digitais do sensor TM/Landsal, nas bandas 1, 2, 3, 4,5 e 7 , da passagem de 10 de outubro de 1985 , referentes à órbita/ponto 219/76A, Carta do Levantamento de Solos SemiDetalhados do Estado de São Paulo - Quadrícula de Campinas na escala 1:100.000 (Figura 1) e o SIG (Sistema de Informação Geográfica) SPRING - Sistema de Processamento de Informação Georeferênciada, desenvolvido pelo Instituto Nacional de Pesquisas Espaciais - INPE.

\section{Área de estudo}

A área de estudo localiza-se no Estado de São Paulo, mais especificamente na região noroeste da cidade de Campinas, e abrange parte dos municípios de Sumaré, Nova Odessa, Monte Mor e Nova Veneza. Segundo Oliveira et al. (1981) trata-se de uma área bastante diversificada quanto aos tipos de solo. A Figura 2 mostra a área de estudo.

Os itens seguintes descrevem os processos usados para a caracterização e extração das feições associadas aos latossolos.

\section{Comportamento espectral dos solos}

Para se poder caracterizar e extrair informações de solos em uma imagem, é necessário que se conheça o seu comportamento espectral e como ele é afetado pelos fatores intrínsecos aos solos. O comportamento espectral dos solos varia de acordo com fatores intrínsecos, dentre os quais têm sido citados na literatura, como os mais importantes, a umidade, a matéria orgânica, a textura, a estrutura, a cor, a capacidade de troca catiônica, o teor de ferro, a mineralogia, a rugosidade superficial e o material de origem (Condit, 1970),
(Beck et al., 1976), (Stoner et al.,1980), (Stnoner \& Baugardener, 1981), (Formaggio \& Epiphanio, 1988), (Galvão, 1994) e (Demattê, 1995).

Esses fatores contribuem decisivamente nas cores em que referidos solos aparecem nas imagens. Os solos lateríticos, especificamente os mais ricos em teor de ferro, aparecem em composição colorida normal em tons de magenta, cor essa devida ao teor de ferro constituinte desses solos e os podzólicos aparecem, em geral, na cor branca.

\section{RESULTADOS E DISCUSSÃO}

\section{Processamento digital da imagem}

A cada etapa que será descrita a seguir, foi feita a comparação dos resultados dos processamentos com a carta de solos da área, de maneira a se analisar o efeito de tais processamentos.

\section{a) Pré-processamento}

O pré-processamento das imagens utilizado neste estudo compõe-se da restauração e do registro das imagens.

A técnica de restauração de imagem (Fonseca,1981) tem por função recuperar a resolução do sistema sensor TM/ Landsat $(30 \mathrm{~m})$ que é alterada (38 ou 40m, por exemplo) durante o processo de aquisição das imagens. Utilizou-se a técnica de restauração para se obter a imagem com sua resolução espacial próxima à resolução efetiva do sistema sensor TM $(30 \mathrm{~m})$. Este processo foi realizado em cada uma das bandas utilizadas no estudo.

O processo de registro foi necessário para sobrepor a carta de solos às imagens digitais processadas.

\section{b) Ampliação linear de contraste}

A ampliação linear de contraste tem por objetivo realçar as feições das imagens, as quais possuem baixo contraste, devido a fatores como a absorção atmosférica, alvos da cena etc.

Para evitar a subjetividade do realce de contraste que varia de pessoa para pessoa, Sousa, 1996, realizou uma análise estatística das imagens, antes e depois da aplicação da técnica, e observou que, trabalhando com os valores de média e desvio-padrão, pode-se eliminar a subjetividade do realce da cena, ou seja, com uma média em torno de 127 e um desviopadrao na faixa de 40 a 50 , ter-se-á bons resultados nesse processo. A ampliação linear de contraste permitiu melhor discrimanação visual dos solos em estudo.

\section{c) Composiçãa colorida}

Em virtude de se haver trabalhado com composições coloridas, foi necessário verificar-se qual seria a melhor composição para este tipo de estudo. Desta forma, Sousa, 1996, realizou tal estudo e verificou que as melhores composições são as formadas pelas bandas TM7, TM5, TM3 e TM2, com as seguintes combinações de banda: $7 \mathrm{R} / 5 \mathrm{G} / 2 \mathrm{~B}$ e 7R/5G/3B. Essas duas composições permiliram melhor associação das respostas vistas nas imagens com as leições 
associados aos solos lateríticos (cor). A Figura 3 mostra a composição colorida $7 \mathrm{R} / 5 \mathrm{G} / 2 \mathrm{~B}$.

\section{d) Transformação IIIS}

O espaço IHS é uma forma alternativa ao espaço RGB para a representação das cores. Tem-se, para o espaço IHS, que a componente I representa a intensidade, a componente H o matiz e a componente S a saturação. Essas componentes podem ser analisadas e manipuladas individualmente, o que não ocorre nas bandas do espaço RGB.

O realce obtido pela ampliação linear de contraste pode ser melhorado a partir das componentes I e S desta imagem no espaço IHS, pois neste caso elimina a interdependência das bandas existentes no espaço RGB (Dutra \& Meneses, 1987). Foi aplicada, na imagem transformada do espaço RGB para o IHS, uma ampliação linear de contraste nas componentes I e S.

As imagens geradas na forma de composição colorida foram submetidas à avaliação de suas contribuições na identificação das feições associadas aos diferentes solos, através da sobreposição dessas imagens com a Carta de Solos da área de estudo.

A composição colorida 7R/5G/2B, selecionada dentre as várias composições segundo (Sousa, 1996), foi submetida à transformação IHS (Figura 4) e ofereceu melhores recursos visuais na individualização das associações dos diferentes solos, especificamente os latossolos, pois é nítida a melhora no realce das cores, quando comparada com a composição antes da transformação.

\section{e) Extração do Latossolo pela cor}

Neste etapa, deseja-se extrair as manchas associadas aos solos lateríticos especialmente aqueles com maior teor de ferro, através do seu atributo cor; portanto, trabalhou-se apenas com a componente $\mathrm{H}$ do espaço IHS. Esta componente identifica o matiz (cor) das feições desses solos. A Figura 2.5(b) mostra a representaçao esquemática da componente $\mathrm{H}$.

Pode-se observar, na imagem da Figura 5 (a), que o solos lateríticos mais ricos em ferro se apresentam na imagem com um intervalo de cores próximo à cor magenta no espaço RGB.

Na Figura 5 (b) que representa a componente $H$, verifica-se, através da leitura de pixel, que as feições associadas aos solos com maior teor de ferro podem ser representadas pelo intervalo de níveis de cinza maiores que 210.

Com o propósilo de se obler uma imagem com esses níveis de cinza, foi realizada uma limiarização na componente $\mathrm{H}$, de forma a se obter uma imagem binária $f(x)$, de acordo com os critérios mostrados na Equação 1.

$$
f(x)=\left\{\begin{array}{l}
0 \quad 0 \leq H(x) \leq 210 \\
255 \quad \text { c.c. }
\end{array}\right.
$$

\section{f) Transformação morfológica}

A Morfologia Matemática possui ferramentas bem adaptadas para a análise espacial de estruturas e utiliza teoria de conjuntos, topologia, reticulados etc, para extrair objetos em uma imagem (Serra, 1982), (Serra, 1986), (Barrera, 1987), (Haralick et al., 1987), (Haralick \& Shapiro, 1991), (Banon \& Barrerra , 1994) e (Barrera et al., 1995).

A Figura 6 mostra os operadores de dilatação $\left(\delta_{B}\right)$ e erosão $\left(\varepsilon_{B}\right)$ de f com relação a um elemento estruturante $\mathrm{B}=[1$ 1.1]. Observa-se que, para o caso da erosão, a curva descrita está sempre abaixo ou no máximo igual ao perfil $\int$. Para o caso da dilatação, a curva descrita está sempre acima ou no mínimo igual ao perfil f.

As transformações $\gamma_{B}$ e $\gamma_{B}$ e $\phi_{B}$ dadas pelas seguintes composições

$$
\gamma_{B}=\delta_{B} \varepsilon_{B} \text { e } \phi_{B}=\varepsilon_{B} \delta_{B}
$$

são chamadas, respectivamente, abertura e fechamento (morfológico). A abertura elimina pequenas regiốes claras da imagem, enquanto o fechamento elimina pequenas regiões escuras. O tamanho das regiões a serem eliminadas é sempre menor que o elemento estruturante escolhido. Combinando-se filtros de abertura e fechamento, tem-se um resultado mais homogeneizado (Frery \& Candeias, 1994).

Neste trabalho utiliza-se um filtro

$$
f_{1}=\phi_{B} \gamma_{B}(f)
$$

para reduzir os ruídos na máscara (imagem binária) $f$. Supõe-se que os objetos a serem eliminados sejam

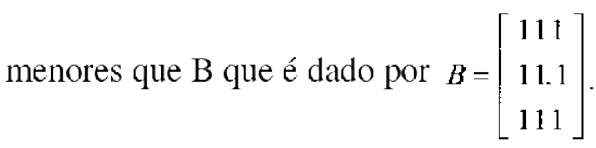

A Figura 5 mostra a composição colorida, a componente $\mathrm{H}$, a componente $\mathrm{H}$ binarizada de acordo com a Equação 1, e esta última imagem após a filltragem morfológica (Equação 3 ).

\section{g) Visualização do latossolo com seu contorno}

Para visualizar o resultado encontrado para identificar o latossolo, desenvolveu-se um programa na linguagem LEGAL (Cordeiro et al., 1996 e Câmara, 1995) que sobrepõe o resultado do contorno dos latossolos obtidos com a imagem colorida.

O contorno é dado por:

$$
f_{2}=f_{1}-\varepsilon_{B}\left(\phi_{B}\right)
$$

Combinando-se o contorno com a imagem colorida, obtém-se a imagem representada pela Figura 7. 


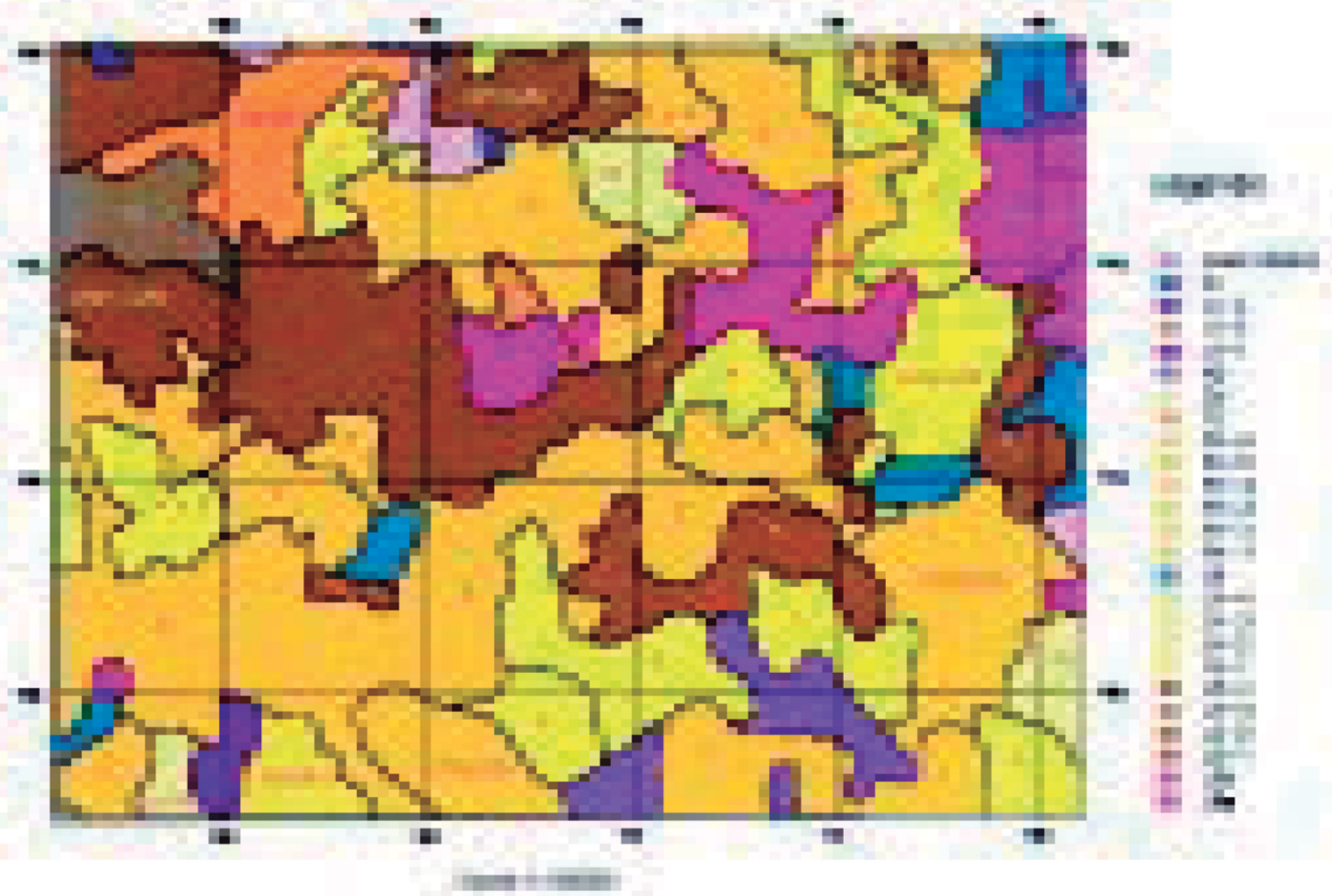

Figura 1. Distribuição dos solos as área de estudo (Fonte: Adaptado de Oliveira et al., 1981).

Latossolo Roxo

LRe - Eutrófico, A moderado. Unidade Ribeirão Preto LRd - Distrófico, A moderado. Unidade Barão Geraldo

Latossolo Vermelho - Escuro

LE-1 - Álico, A moderado, textura argilosa. Unidade Limeira

LE-2 - Álico, A moderado, textura argilosa. Unidade Santo Antonio

Álico, A proeminente, textura média ou argilosa Unidade Cillos

LE-4 - Álico, A moderado, textura média. Unidade Hortolândia

Latossolo Vermelho - Amarelo

LV-1 - Álico, A moderado, textura argilosa. Unidade Mato Dentro LV-2 - Álío, A proeminente, textura média. Unidade Speculas

LV-3 - Álico, A moderado, textura média. Unidade Laranja Azede

LV-4 - Álico, A moderado, textura média, Unidade Bela Aliança

Latossolo Vermelho - Amarelo Húmico

LH. - Álico, textura média ou argilosa. Unidade Camarguinho.

Podzólizo Vermelho - Amarelo

PV-1 - Abruptivo, A moderado espesso, textura arenosa/médla. Unidade Alva PV-2 - A moderado ou proeminente, textura arenosa/médla Unidade Usina

PV-2 - A moderado ou proeminente, textura arenosa/media
PV-3 - A moderado, textura argilosa. Unidade Holambra

PV-5 - A moderado, textura média ou argilosa. Unidade Palmeiras

PV-6 - A moderado, textura argilosa. Unidade Valinhos

Terra Roxa Estruturada

TE - Eutrófica ou distrófica, A moderado. Unidade estruturada

Cambissolo

$\mathrm{Cb}$ - A moderodo, distrófico, textura média. Unidade Palha

Solos Areno - Quartzosos Podzólicos Profundos

AQ - Solos areno - quartzosos podzólicos profundos, distróficos. Unidade Panorama

Solos Litólicos

Li-I - A moderado, substrato sedimentos da formação Tubarão

$\mathrm{Li}-2$ - A moderado, substrato basalto

LI-3 - A moderado, substrato granito - gnaisse

Complexo Indiscriminado de Solos Hidromórficos

Hi - Gley Pouco Húmico e Gley Húmico

\section{Associação de Solos}

Designada pelo conjunto de símbolos das unidades taxonômicas componentes em ordem decrescente de ocorrência e coloridos com cor da unidade predominante. Ex: LRd+LRe+LE-1 (Unidade Barão Geraldo + Unidade de Ribeirão Preto + Unidade Limeira).

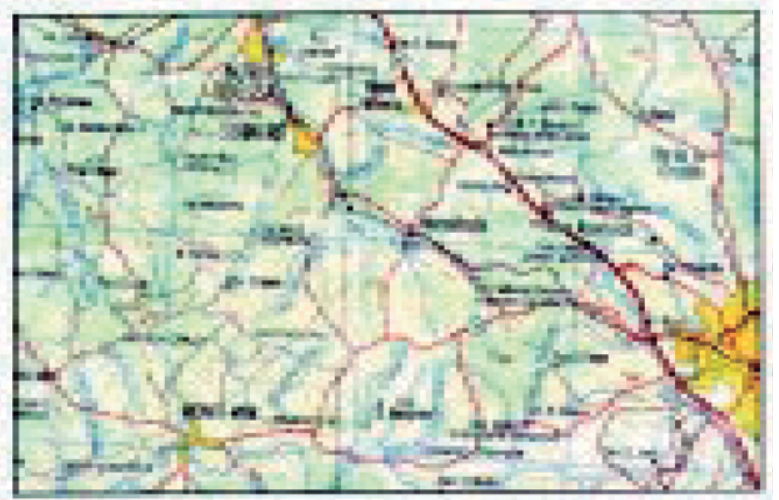

Figura 2. Carta topográfica da área de estudo (Fonte: Carta topográfica de Campinas, 1975).

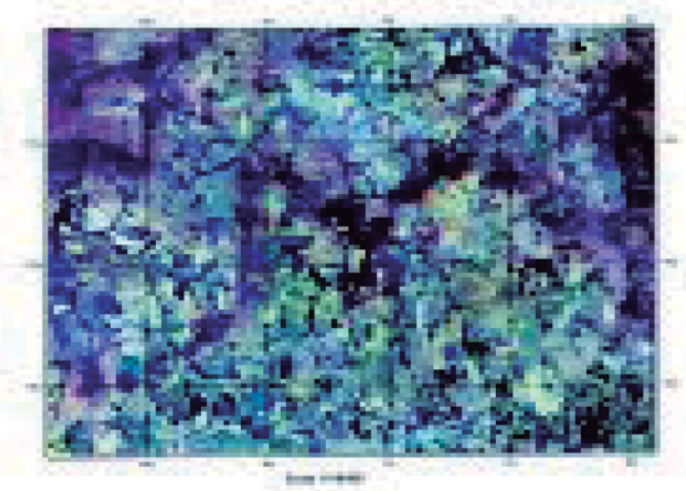

Figura 3. Composição colorida 7R/5G/2B com a sobreposição da distribuição de solos da área de estudo. (Fonte: Sousa, 1996). 


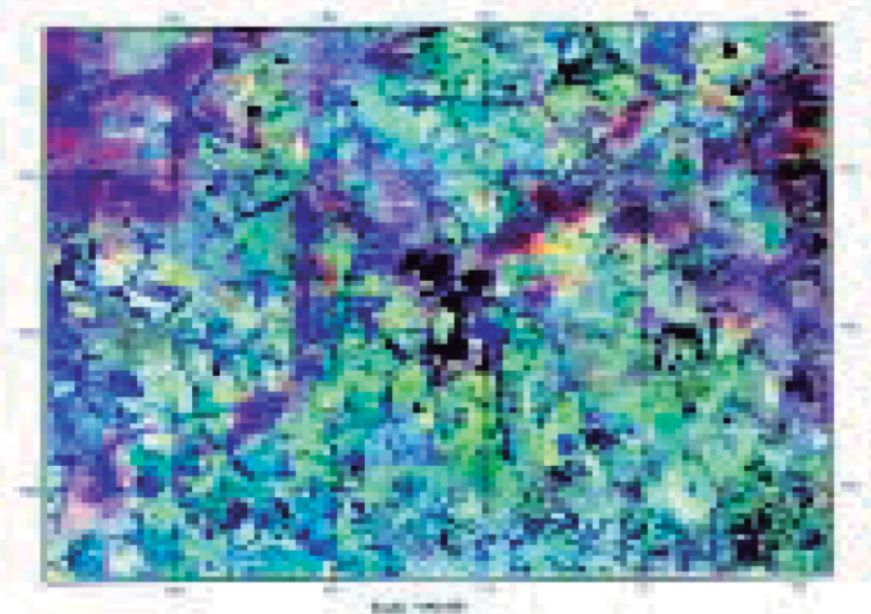

Figura 4. Transformação I H S da composição colorida 7R/5G/2B (Fonte: Sousa, 1996).
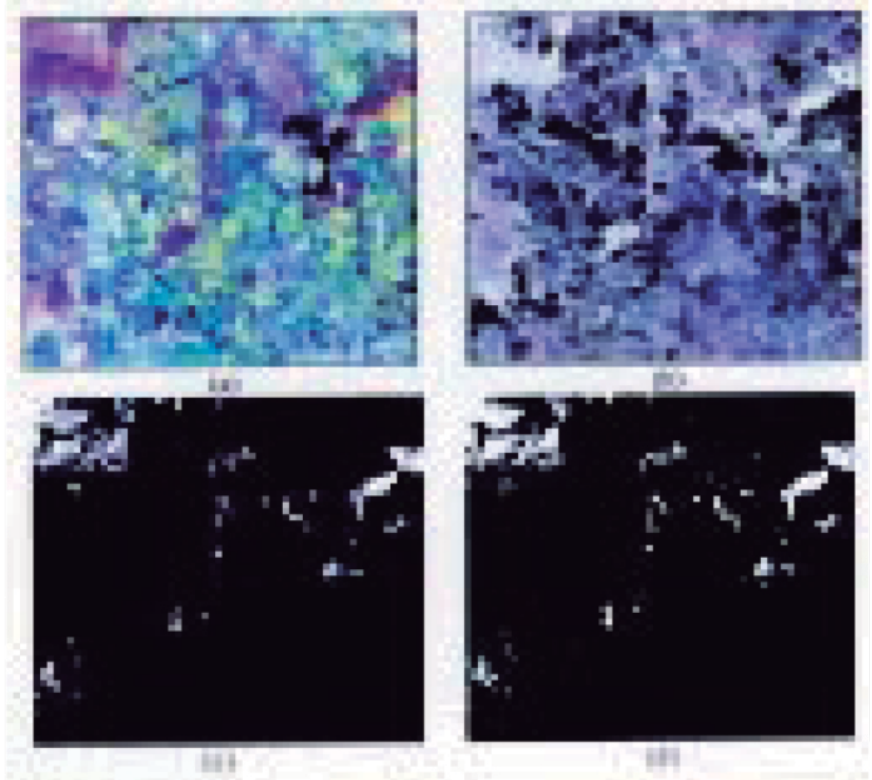

Figura 5. a) Composição colorida. b) componente H. c) componente $H$ binarizada d) imagem após filtragem morfológica.

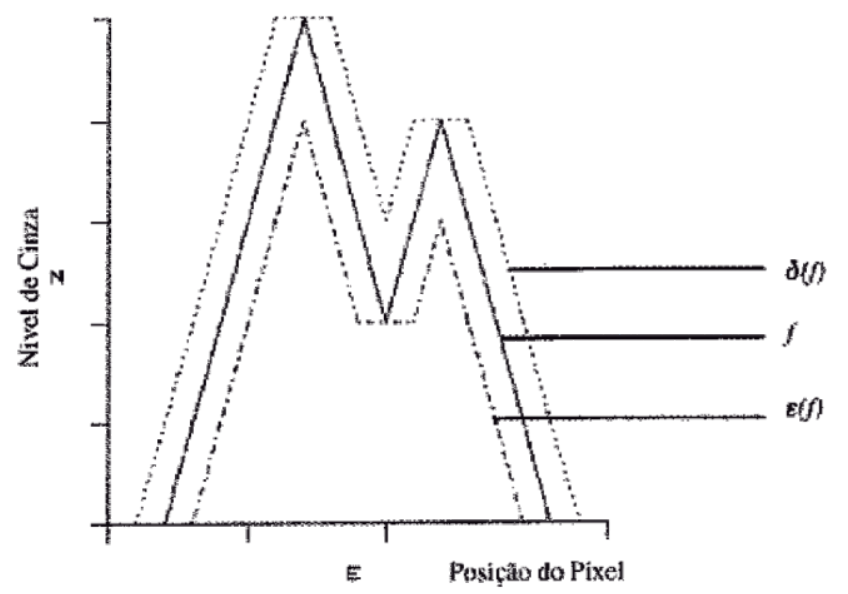

Figura 6. Erosão e dilatação em uma linha de por $\mathrm{B}=11.11$.

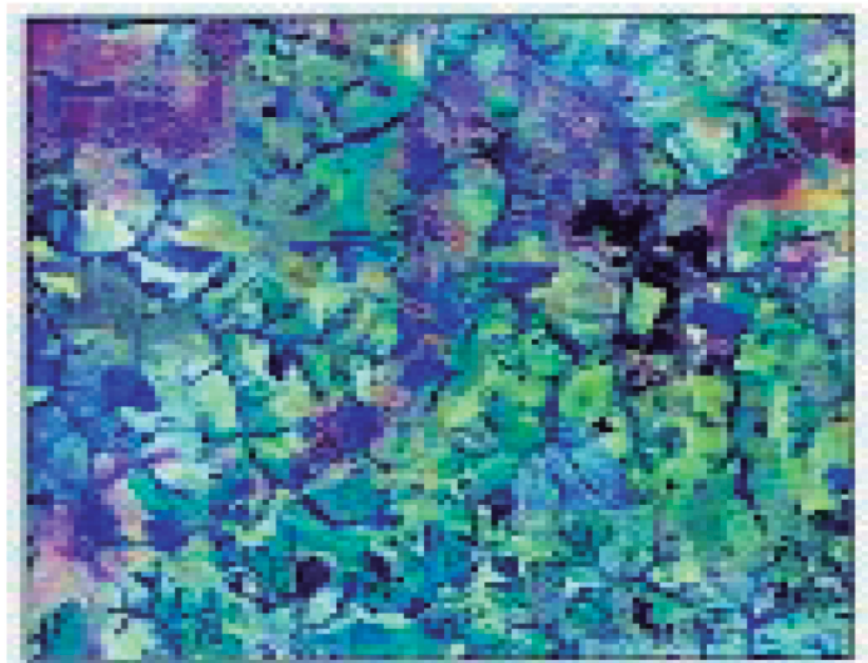

Figura 7. Extração das manchas dos solos com maior teor de ferro.

\section{CONCLUSÕES}

1. Pelos resultados apresentados verifica-se que as técnicas de processamento digital de imagens (restauração, realce linear de contraste e transformação IHS) serviram aos propósitos do trabalho permitindo, desta forma, que as feições associadas às diferentes unidades de mapeamento de solo, e especificamente os solos lateríticos com maior teor de ferro, pudessem ser identificadas e realçadas podendo, assim, auxiliar no processo de levantamento dos solos. Além deste processo de identificação foi possível gerar um produto georeferênciado com os contos das manchas desses solos permitindo, então, se dispor de material semelhante ao de uma carta, que pode ser utilizada em várias etapas do processo de levantamento de solos como mais uma ferramenta de auxílio para este tipo de estudo.

2. Este estudo mostrou, ainda, que é possível se obter uma classificação automática para esta área, baseada em atributos de cor e tamanho.

3. As vantagens de se mapear os latossolos automaticamente são dadas pela otimização de custo, com análise visual, minimização dos erros inerentes ao mapeamento, redução de visitas de campo etc.

\section{REFERÊNCIAS BIBLIOGRÁFICAS}

BANON G.J.F. E BARRERA J. Bases da Morfologia Matemática para a análise de imagens binárias. IX Escola de Computação, Recife, 24 -31, julho, pp. 230, 1994.

BARRERA J. Uma abordagem unificada para os problemas de Processamento Digital de Imagens: a Morfologia matemática. São José dos Campos: INPE, 1987. (Dissertação de mestrado)

BARRERA J., BANON G.J.F. E LOTUFO R.A. A mathematical morphology toolbox for the KHOROS system: specifications for Version 1.2b. In: Workshop'95 de Morfologia matemática, São José dos Campos. 1995, Anais, São José dos Campos . INPE, 1995. Disponível: site inpe. URL http : // www . inpe. br/ rbanon/URLib2/col/ime.usp.br/jb/1996/04.03.14.02. Consultado em dez. 1995. 
BECK R.H., ROBINSON B.F., MOFEE W.H., PETERSON J.B. Spectral characteristics of soils related to the interaction of soil moisture, organic carbon and clay content. West Lafayette, IN. Purdue University, (LARS Information Note 081176 ), 1976.

CÂMARA G. Modelos, Linguagens e Arquiteturas para Bancos de dados Geográficos. São José dos Campos: INPE, 1995. (Tese de doutorado em Computação Aplicada).

CANDEIAS A.L B. Aplicação da Morfologia Matemática à Análise de Imagens de Sensoriamento Remoto. São José dos Campos: INPE, 1995. (Tese de doutorado em (Computação $\Lambda$ plicada).

CONDIT H.R. The spectral reflectance of americam soils. Photogrammetric Engineering, Falls Church, 36:955$966,1970$.

CORDEIRO J.P., AMARAL S., FREITAS U. M., CÂMARA G. Álgebra de Geo-Campos e suas Aplicações. In: Simpósio Brasileiro de Sensoriamento Remoto, 8., Salvador, 1996, Anais. São Paulo:INPE/SELPER, 1996. 6p. CD ROM, Scção artigos, p. 348-354.

DÊMATTE J.A.M. Relações entre dados espectrais e características físicas, químicas e mineralógicas de solos desenvolvidos de rochas eruptivas. Piracicaba: ESALQ, 1995. 265p. (Tese de doutorado).

DUTRA, L.V.; MENESES, P. R. Realce de cores em imagens de sensoriamento remoto utilizando-se rotação de matiz no espaço IHS. São José dos Campos, INPE-4207-PRE/ 1088, 1987.

FONSECA, L.M.G. Restauração e interpolação de imagens do satélite Landsat por meio de técnicas de projetos de filtro FIR. São José dos Campos: ITA, 1988. (Dissertação de Mestrado).

FORMAGGIO A.R., EPIPHNANIO J.C. Reflectance pattern from brazilian oxisols and their physical and chemical characteristics. Proceedings of the 16 International Congress of International Society Photogrammetry and Remote Sensing, Kyoto, Japan, ISPRS, p. 497-505, v. 27, 1988.

FRERY, A.C.; CANDEIAS , A.L.B. On the improvemnet of 1-look SAR image segmentations with mathematical morphology. São José dos Campos, INPE. 1994. (INPE5577-PRE/18(05).
GALVÃO L.S. Litoestratigralia da refletância espectral e uma abordagem quantitativa para análise de espectros. São Paulo: USP/ Instituto Astronômico e Geofísico, 1994. 179p. (Tese de doutorado em Geofísica.

HARALICK R.M., STERNBERG S.R., ZHUANG X. Image analysis using mathematical morphology, IEEE Pattern Anal. Machine Intell., vol. PAMI-9, no. 4, pp. 532-555, Jul., 1987.

HARALICK R.M., SHAPIRO L. G. Computer and robot vision, vol. 1, New York, Addison Wesley, 1991.

OLIVEIRA J.B., MENK J.R F., BARBIERI J.L., ROTTA E.L., TREMOCOLDI W. Levantamento pedológico semidetalhado do estado de São Paulo. Campinas, EMBRAPA/SECRETARIA DA AGRICULTURA E A BASTECIMENTO DO ESTADO DE SÃO PAUO, 1981. Folha SF-23-Y-A-II, Quadrícula de Araras, Escala de 1:100.000

RASURE J., ARGIRO. D., SAUER. T., WILLIAMS. C. Visual Language and Software Development Environment for Image Processing. International Journal of Imaging Systems and Technology, (2), pp. 183-199, 1990.

SERRA J. Image Analysis and Mathematical Morphology. Volume 1. Academic Press, London, 610 p., 1982.

SERRA J. Introduction to mathematical morphology. Computer Vision. Graphics and Image Processing, vol. 35, no.3, pp. 283-305, 1986.

SOUSA E.B. Técnicas de Processamento de Imagens Aplicadas à Localização de Solos Lateríticos. São .José dos Campos: ITA, 1996. (Dissertação de mestrado).

STONER E.R., BAUGARDENER M. F. Characteristic variations in reflectance of surface soils. Soil Science. Am. Journal, 45, 1161-1165, 1981.

STONER E.R, BAUMGARDNER M. F., BIEHL L.L., ROBINSON B. F. Atlas of soil refletance proprieties. West Lafayette, Purduc University, Indiana, Agricultural Experimental Station, 75p., 1980 (Research Bulletin 962).

VERTAMATTI E. Tecnologia de solos tropicais. $\Lambda$ postila do curso de pós-graduação $\mathrm{cm}$ Engenharia de Infra-

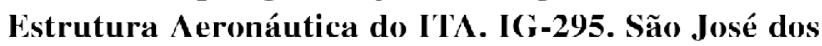
Campos, São Paulo, 1994. 